\title{
Determining the Chirality of Néel-type Magnetic Skyrmions by Phase Retrieval with Four-dimensional Lorentz Scanning Transmission Electron Microscopy
}

\author{
Zhen Chen ${ }^{1}$, Teng $\mathrm{Xu}^{2}$, Gregory Fuchs ${ }^{1}$, wanjun Jiang ${ }^{2}$ and David Muller ${ }^{1}$ \\ ${ }^{1}$ Cornell University, Ithaca, New York, United States, ${ }^{2}$ Tsinghua University, Beijing, Beijing, China \\ (People's Republic)
}

Imaging magnetic fields with an electron microscope relies on detecting a spatially nonuniform phase shift of the electron wave induced by the lateral magnetic induction field $\left(\boldsymbol{B}_{x y}\right)$ in the sample. However, the lateral magnetic induction fields for ferromagnets are not always parallel to the magnetization when strong stray fields are present. This is the case for Néel-type domain wall or skyrmion, where a zero lateral magnetic induction is expected for normal incidence illumination. As reported by Lorentz TEM (LTEM) $[1,2]$, Néel-type domain walls or skyrmions can only be seen in a tilted sample condition. The required tilting, however, results in increasingly delocalized magnetic induction, which makes it more challenging to image Néel-type than Bloch-type skyrmions. As a practical matter, the spatial resolution of LTEM is degraded to tens of nanometers due to the delocalization from the large defocus needed for sufficient contrast. Therefore, it is difficult to identify the internal spin structures of skyrmions with LTEM, especially below the sub-100 $\mathrm{nm}$ length scale.

Differential phase contrast (DPC) imaging in Lorentz scanning TEM (LSTEM) has been used for imaging magnetic domain walls since 1970s [3]. It uses a focused probe and can potentially reach atomic resolution [4]. Furthermore, it directly measures the beam deflection angle, or equivalently the gradient of the phase shift, by the local magnetic induction field in the sample. This is straightforward for interpretation and quantification. However, the lack of low-noise detectors made DPC for magnetic imaging less widely adopted than LTEM. The situation is changing now, with the new generation of fast pixelated detectors enabling four-dimensional LSTEM (4D-LSTEM) (schematic in Figure 1(a)) and making DPC much more flexible and efficient [5]. Here, using a high-dynamic-range electron microscopy pixel array detector (EMPAD) developed at Cornell University [6], we show that ultrahigh angular precision, $\sim 0.1 \mu \mathrm{rad}$, can be achieved [7]. The high precision enables direct imaging of the internal structures of Bloch-type skyrmions including the topology and lateral discontinuities (Figure 1(b)). It also allows us to image magnetic domain structures in embedded cobalt films with magnetic layers only $\sim 4$ atoms thick.

Figure 2 shows the experimental LSTEM images of Néel-type skyrmions from a multilayer film with $\mathrm{CoTb}$ ferrimagnetic layers $\left(\left[\mathrm{MnSn}_{3} / \mathrm{CoTb} / \mathrm{SiN}_{\mathrm{x}}\right] 20\right), B_{x}$ in Figure 2(a) shows a quadrupole-like distribution, which agrees well with the simulation in Figure 1(c). Figure 2(c) shows the intensity along the diagonal directions, marked by the red dashed lines in Figure 2(b). The intensity asymmetry for the two peaks agrees with the simulation from a skyrmion with a right-handed chirality. We also confirm that the height of the two peaks in $B_{x}$ is switched in skyrmions with a left-handed chirality in a film with reversed layer sequence. Our results thus confirm that the spin chirality of Néel-type skyrmions can be directly determined using 4D-LSTEM. [9] 

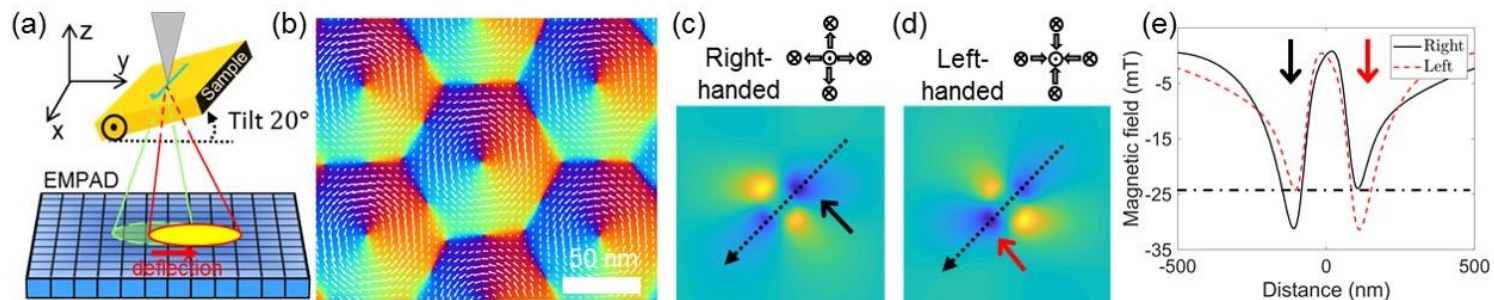

Figure 1. Chirality determination for a Néel-type skyrmion via 4D-LSTEM. (a). Schematic of 4D-LSTEM with a tilted sample and an EMPAD; (b) Magnetic field of Bloch-type skyrmion lattice in FeGe measured at $94 \mathrm{~K}$. (c) \& (d). Simulations of $\mathrm{x}$-component of magnetic induction field (Bx) of a Néel-type skyrmion (diameter $80 \mathrm{~nm}$ ) with right-handed (c) and left-handed (d) chirality. (e). Magnitude of Bx along the dashed line on (c) and (d). The sample is tilted along the horizontal (x) axis clockwise when looking along positive $\mathrm{x}$-axis towards right.
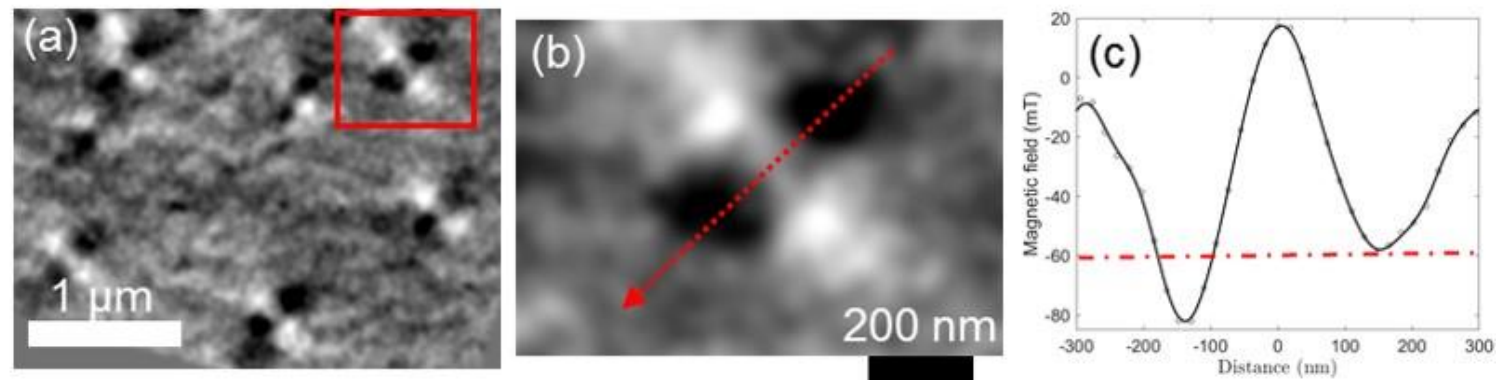

Figure 2. Experimental magnetic induction field and chirality determination for Néel-type skyrmions in a multilayer metallic film [Mn3Sn/CoTb $(6 \mathrm{~nm}) / \mathrm{SiNx}] 20$ grown on Si3N4 membrane. (a). x-component of magnetic induction field of Néel-type skyrmions (Bx). (b). Zoomed in for $\mathrm{Bx}$ in the rectangular region on (a). (c). Magnitude of Bx along the dashed lines on (b).

\section{References}

[1] MJ Benitez, et al., Nature Communications, 6 (2015), p. 8957.

[2] SD Pollard, et al., Nature Communications, 8 (2017), p. 14761.

[3] J Chapman, et al., Ultramicroscopy, 3 (1978), p. 203-214.

[4] M Edström, et al., Physical Review B, 99 (2019), p. 174428.

[5] M Krajnak, et al., Ultramicroscopy, 165 (2016), p. 42-50.

[6] MW Tate, et al., Microscopy and Microanalysis 22 (2016), p. 237.

[7] KX Nguyen, et al., arXiv:2001.06900 (2020).

[8] W Jiang, et al., Physical Review B, 99 (2019), p. 104402.

[9] Research supported by US NSF DMR-1539918, DMR-1719875 and US DARPA TEE-D18AC00009. 